\title{
Electronic band gaps and transport in aperiodic graphene superlattices of Thue-Morse sequence
}

\author{
Tianxing $\mathrm{Ma}^{1,2, *}$, Chun Liang ${ }^{2}$, Li-Gang Wang ${ }^{3,2, \dagger}$ and Hai-Qing Lin $^{2}$ \\ ${ }^{1}$ Department of Physics, Beijing Normal University, \\ Beijing 100875, China \\ ${ }^{2}$ Beijing Computational Science Research Center, \\ Beijing 100084, China \\ ${ }^{3}$ Department of Physics, Zhejiang University, \\ Hangzhou 310027, China
}

\begin{abstract}
We have studied the electronic properties in aperiodic graphene superlattices of Thue-Morse sequence. Although the structure is aperiodic, an unusual Dirac point (DP) does exist and its location is exactly at the energy corresponding to the zero-averaged wave number (zero- $\bar{k})$. Furthermore, the zero- $\bar{k}$ gap associated with the DP is robust against the lattice constants and the incident angles, and multi-DPs may appear under the suitable conditions. A resultant controllability of electronic transport in Thue-Morse sequence is predicted, which may facilitate the development of many graphene-based electronics.
\end{abstract}

Graphene has attracted enormous attention of experimentalists and theorists [1-8] since its discovery. The interest is driven by its potential technological applications and unconventional low-energy behavior, since graphene has a unique band structure with the conductance and valance bands touching at Dirac point (DP). Recently, scientists anticipate that graphene-based optoelectronics may supplement silicon-based technology, which is nearing its limits [9]. For superlattices are vastly successful to control the electronic transport [10], to facilitate the application of graphene-based devices, the graphene superlattices (GSLs) with electrostatic potential or magnetic barrier have also received broad focuses of theoretical and experimental investigations 11 20]. In such GSLs, the new DP appears in the band structures [15, 16] and it's exactly located at the energy corresponding to zeroaveraged wave number (zero- $\bar{k}$ ) [17]. Contrary to Bragg gaps, the zero- $\bar{k}$ gap associated with the new DP is insensitive to both the lattice constant and the structural disorder, resulting in better controllability of electronic transport in GSLs. Most recently, Zhao and Chen predicted a controllable electron transport in a Fiboncacci quasi-periodic GSL [18].

In this letter, we investigate the electronic band gaps and transport in the graphene-based Thue-Morse (TM) sequence. As a typical aperiodic system, the TM lattice has been widely studied 21 26], which is known to have a singular continuous Fourier transform 22 and a Cantorlike phonon spectrum [23], and it is more "disordered" than the Fibonacci sequence [21, 22]. The TM lattice has a deterministic geometry structure, and its electronic properties are also of great interesting [24]. In the stud-

\footnotetext{
*txma@bnu.edu.cn

†sxwlg@yahoo.com.cn
}

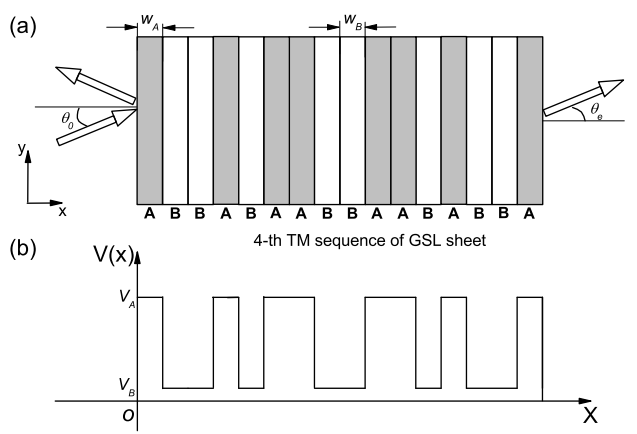

FIG. 1: (Color online) (a) Example of a 4-th order GSL TM sequence. (b) The schematic profiles of the potentials $V_{A}$ and $V_{B}$ corresponding to (a).

ied graphene-based TM sequence, we find that zero- $\bar{k}$ gap and DP do exist, which results in robust electronic transport properties. Moreover, the splitting behavior of the passing bands in the graphene-based TM sequence is very different from that in the graphene-based Fibonacci sequence, which leads to the different electronic transport property.

For an $n$-th TM sequence, $S(n)$, it contains elements $A$ and $B$, and follows the inflation rule: $A \rightarrow A B, B \rightarrow$ $B A$ from generation to generation with $S(1)=A B$ (for example, see Ref. 27] ). Let $S_{1 / 2}^{L}(n)$ and $S_{1 / 2}^{R}(n)$ denote the left and right half parts of an $n$-th order TM sequence, respectively, then iteration relation for any $(n+1)$-th TM sequence is written as

$$
S(n+1)=S(n) S_{1 / 2}^{R}(n) S_{1 / 2}^{L}(n) .
$$

Naturally, $S(2)=A B B A, S(3)=A B B A B A A B$, and so on. In our cases, $A(B)$ denotes barrier $V_{A}\left(V_{B}\right)$ with its width $w_{A}\left(w_{B}\right)$. Fig. 1 shows the schematic illustration of the 4th order GSL TM sequence and the corresponding distributions of barriers and wells.From Eq. (1) and Fig. 

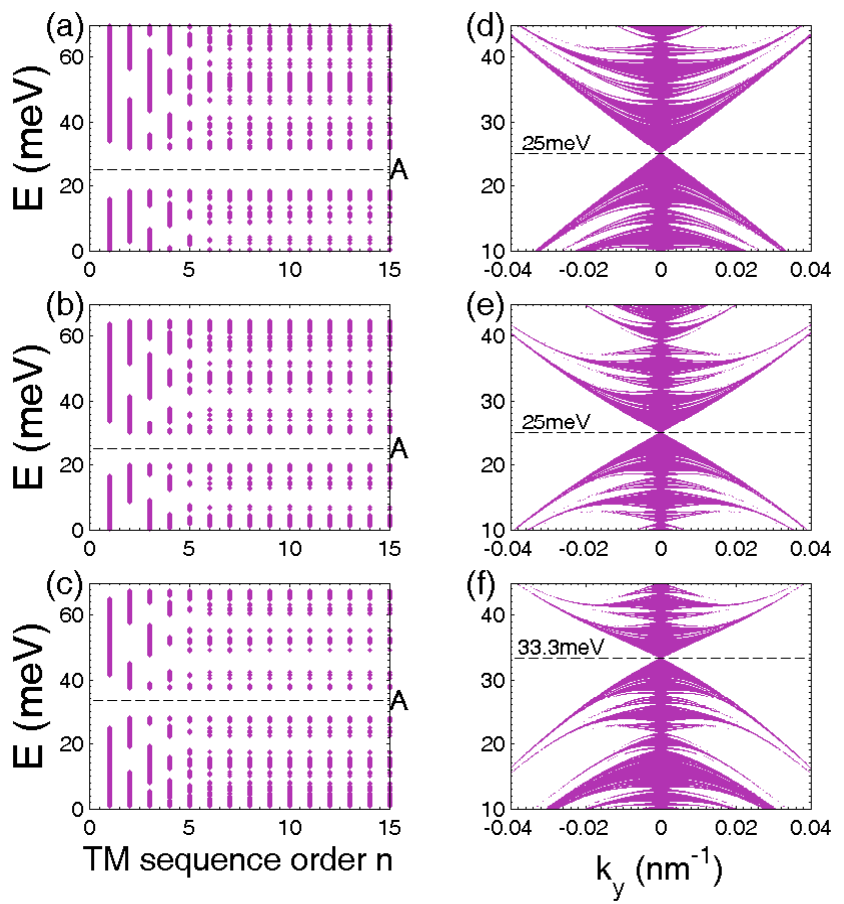

FIG. 2: (Color online) Trace-maps for GSL TM sequences of variable order $n$ under (a) $w_{A, B}=20 \mathrm{~nm}$, (b) $w_{A, B}=25 \mathrm{~nm}$ and (c) $w_{A}=2 w_{B}=40 \mathrm{~nm}$ with $V_{A}=50 \mathrm{meV}, V_{B}=0$, and $k_{y}=0.015 \mathrm{~nm}^{-1}$. (d), (e) and (f) are band structures for $n=8$ corresponding to (a), (b) and (c), respectively. The horizontal dashed black line denotes the new Dirac point's location, and the white areas are band gaps and the solid areas are passing bands.

1 , it is readily to know that the number of $A, N_{A}$, equals to that of $B, N_{B}$, in any $S(n)$, i.e., $N_{A}=N_{B}=2^{n-1}$.

Meanwhile the charge carriers near the $\boldsymbol{K}$ point in graphene (near the Fermi level) are described by the Hamiltonian: $\widehat{H}=v_{F} \widehat{\sigma} \cdot \widehat{\mathbf{p}}+V(x) \widehat{I}$, where $V(x)$ is a barrier or well, $v_{F} \approx 10^{6} \mathrm{~m} / \mathrm{s}$ is the Fermi velocity, $\widehat{\sigma}=\left(\widehat{\sigma}_{x}, \widehat{\sigma}_{y}\right)$ are Pauli matrices, and $\widehat{I}$ is a $2 \times 2$ unit matrix. The solution of $\widehat{H}$, acting on the electronic pseudospin wavefunctions, leads to a transfer matrix [17]

$$
M_{j}\left(\Delta x, E, k_{y}\right)=\left(\begin{array}{cc}
\frac{\cos \left(q_{j} \Delta x-\theta_{j}\right)}{\cos \theta_{j}} & i \frac{\sin \left(q_{j} \Delta x\right)}{\cos \theta_{j}} \\
i \frac{\sin \left(q_{j} \Delta x\right)}{\cos \theta_{j}} & \frac{\cos \left(q_{j} \Delta x+\theta_{j}\right)}{\cos \theta_{j}}
\end{array}\right),
$$

which connects the wave functions at $x$ and $x+\triangle x$ inside the $j$ th potential with $\theta_{j}=\arcsin \left(k_{y} / k_{j}\right)$, here $k_{y}$ is the $y$ component of $k_{j}$, and $q_{j}=\operatorname{sign}\left(k_{j}\right) \sqrt{k_{j}^{2}-k_{y}^{2}}$ for $k_{y}^{2}<k_{j}^{2}$, otherwise $q_{j}=i \sqrt{k_{y}^{2}-k_{j}^{2}}$ for $k_{y}^{2}>k_{j}^{2}$. The electronic transmission coefficient $t\left(E, k_{y}\right)$ in such devices can be obtained by [17]

$$
t\left(E, k_{y}\right)=\frac{2 \cos \theta_{0}}{\left(x_{22} e^{-i \theta_{0}}+x_{11} e^{i \theta_{e}}\right)-x_{12} e^{i\left(\theta_{e}-\theta_{0}\right)}-x_{21}},
$$

where $\theta_{0}\left(\theta_{e}\right)$ is the incident (exit) angle, and $x_{i j}(i, j=$ $1,2)$ is the element of $\mathbf{X}[S(n)]=\prod_{j=1}^{N} M_{j}\left(w_{j}, E, k_{y}\right)$, which is the entire transfer matrix of a TM sequence. If we let $x_{n}=\operatorname{Tr}\{\mathbf{X}[S(n)]\}$, it is easy to derive the iteration relation for the trace map of the $n$-th GSL TM sequence as follows 27,29$]$ :

$$
x_{n}=x_{n-2}^{2}\left(x_{n-1}-2\right)+2 .
$$

Treating an $n$-th TM sequence as a unit cell, from Bloch's theorem, we have $\cos \left(\beta_{x} \Lambda_{n}\right)=x_{n} / 2$, where $\Lambda_{n}=N_{A} w_{A}+N_{B} w_{B}$. From Eq.(44), we can calculate the change of the trace map as a function of the order $n$.

In Fig. 2 (a) and (b), we plot the trace maps for two kinds of graphene-based TM sequences with the change of $n$, at the incident angle $\theta_{0}=10^{\circ}$. We take $w_{A, B}=20 \mathrm{~nm}$ in Fig. 2(a), and $w_{A, B}=25 \mathrm{~nm}$ in Fig. 2(b). We find that the passing bands are split into more and more sub-bands as $n$ increases; when $n \geq 6$, the band structures almost become the discontinuous bands. However, we note that the center positions of the Gap A in Figs. 2(a) and 2(b) are the same, and the positions of other gaps are shifted with the change of the lattice constant. Corresponding to Fig. 2(a) and 2(b), the electronic band structures for a TM sequence of $n=8$ have been shown in Fig. 2(d) and 2(e), respectively. It is seen that a new DP appears inside the Gap A and this new DP actually locates at

$$
\bar{k}=\sum_{j=1}^{N} k_{j} w_{j} / \sum_{j=1}^{N} w_{j}=0 .
$$

This condition is valid for periodic and aperiodic graphene superlattices [16 18]. Since $N_{A}=N_{B}$ in our cases, it is easily to obtain the energy for $\bar{k}=0$ as follows:

$$
E=\frac{V_{A}+V_{B} \cdot w_{B} / w_{A}}{1+w_{B} / w_{A}} .
$$

This condition is different from the case of the Fibonacci sequence, which depends on the ration of numbers of layer A and B [18]. Actually, our formula (5) is valid for the condition of the new DP in the graphene-based Fibonacci sequence. Here we would like to emphasize that for the TM sequence, the location of the DP is independent of order $n$; while for the Fibonacci sequence it changes for different order $n$. As an example for the TM sequence, in cases of Fig. 2(a,b,d,e), it is given by $E=25 \mathrm{meV}$ since $w_{A}=w_{B}$.

Since the center of the Gap A in Fig. 2 is located at zero- $\bar{k}$, which is denoted by a dashed black line in Fig. 2 (a) and 2(b), we may call it as the zero- $\bar{k}$ gap. According to Eq.(6), the position of the zero- $\bar{k}$ gap for the TM sequences is not shifted with the lattice constant itself, but is shifted with the change of the ratio of $w_{A} / w_{B}$. As shown in Fig.2(c) and (f), the position of the zero- $\bar{k}$ gap and the DP move to $E \approx 33.33 \mathrm{meV}$ when $w_{A} / w_{B}=2$. 


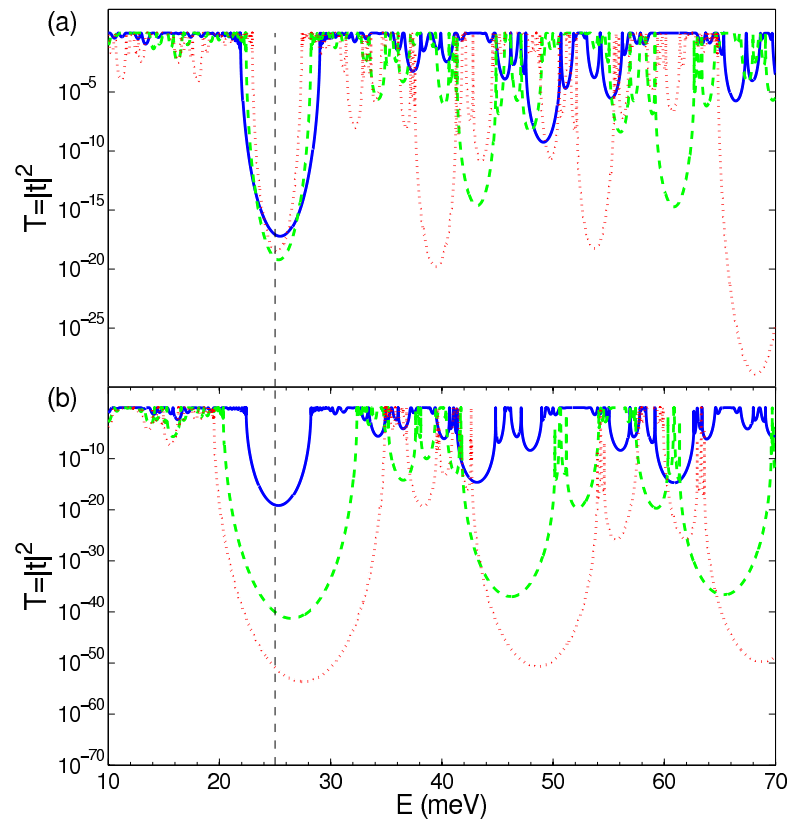

FIG. 3: (Color online) (a) Dependence of transmission spectrum (at $\theta_{0}=10^{\circ}$ ) on different widths of barriers and wells, here solid blue line for $w_{A}=w_{B}=15 \mathrm{~nm}$, dashed green line for $w_{A}=w_{B}=20 \mathrm{~nm}$, and dotted red line for $w_{A}=w_{B}=25 \mathrm{~nm}$; (b) dependence of transmission spectrum on different incident angles: $\theta_{0}=10^{\circ}$ (solid blue line), $20^{\circ}$ (dashed green), and $25^{\circ}$ (dotted red) with fixed lattice constants $w_{A}=w_{B}=20 \mathrm{~nm}$. Other parameters are $V_{A}=50 \mathrm{meV}$ and $V_{B}=0$ for an 8-th GSL TM sequence with total 256 layers.

Fig.3 (a) shows the effect of the lattice constants on the electronic transmission spectrum. Based on Fig. 3(a), it is obvious that the zero- $\bar{k}$ gap is insensitive to the lattice constants themselves. However, the positions of other gaps and passing bands with higher energy are highly dependent on the lattice parameters. From Fig. 3(b), one can also find that the position of zero- $\bar{k}$ gap is weakly dependent on the incident angle $\theta_{0}$, while other Bragg gaps change sensitively with $\theta_{0}$.

Furthermore, one has known that the multi-Diracpoints could appear in the GSLs with periodic potential structures [16, 17]. Here we point out that the extra Dirac points, located at $k_{y} \neq 0$, could also emerge in the GSL TM sequence as the lattice constant increases. See Figs. 4(a) and (b), the slope of the band edges near the DP gradually turns smaller as $w_{A}\left(w_{B}\right)$ increases from $20 \mathrm{~nm}$ to $40 \mathrm{~nm}$. When $w_{A}\left(w_{B}\right)$ is larger than $40 \mathrm{~nm}$, as that shown in Fig. 4 (c), the additional Dirac points appear at the same energy. From Figs. 4(a) to 4(c), we may also find the appearance of additional Dirac points associating with the variation of the zero- $\bar{k}$ gap. In Fig. 4 (d), we show the energy band gaps as a function of the lattice constants in the case of $w_{A}=w_{B}$. In the process of the appearance of the additional Dirac points, the zero- $\bar{k}$ gap opens and closes oscillationally while the
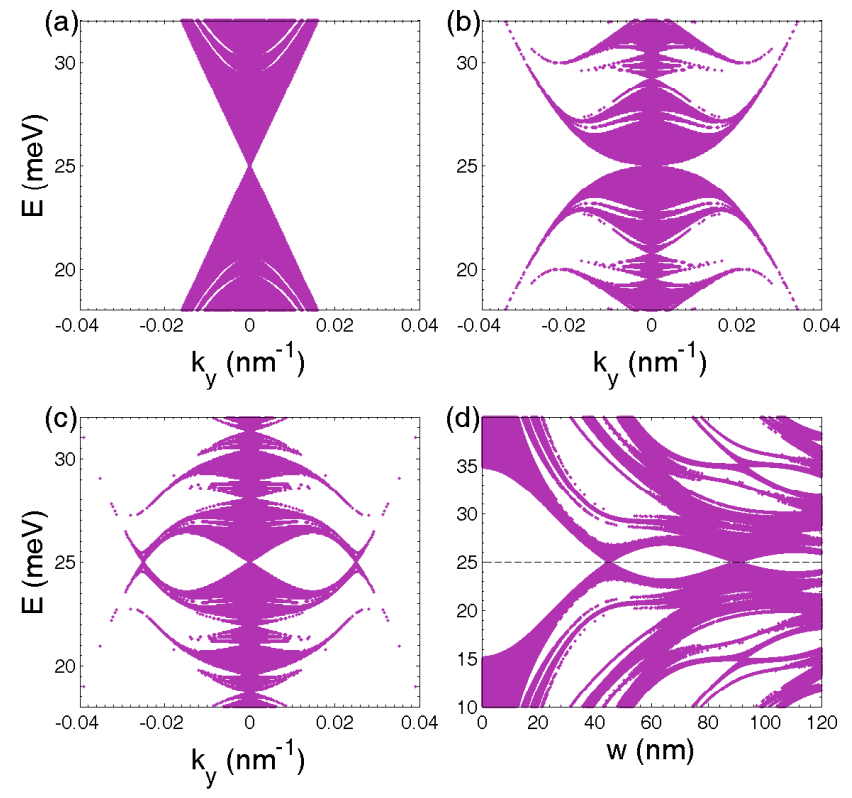

FIG. 4: (Color online) Electronic band structures for (a) $w_{A}=w_{B}=20 \mathrm{~nm},(\mathrm{~b}) w_{A}=w_{B}=40 \mathrm{~nm}$ and (c) $w_{A}=$ $w_{B}=55 \mathrm{~nm} ;$ (d) band gaps depending on the lattice constant $w$ with fixed $k_{y}=0.015 \mathrm{~nm}^{-1}$, here $w_{A}=w_{B}=w$. Other parameters are the same as those in Fig.2.
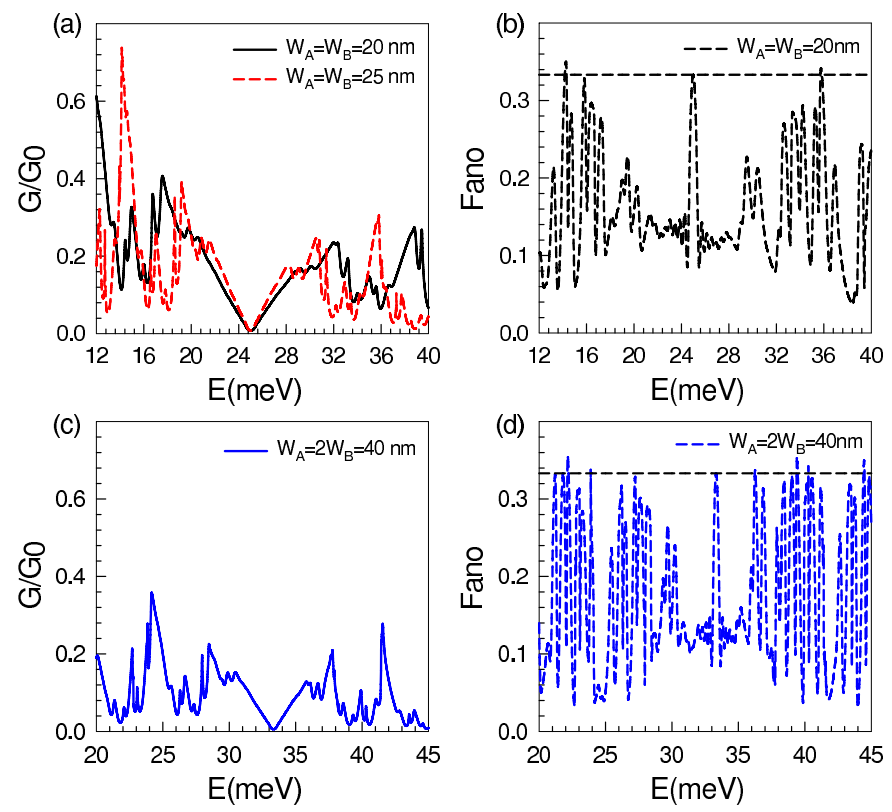

FIG. 5: (Color online) Conductance [(a) and (c)] and Fano factor [(b) and (d)] vs Fermi energy in an 8-th GSL TM sequence. Other parameters are the same as those in Fig.2.

other gaps are shifted greatly.

Finally, we calculate the total conductance $G$ [30] and the Fano factor $F$ [31] in GSL TM sequences, which are given by $G=G_{0} \int_{0}^{\pi / 2} T \cos \theta_{0} d \theta_{0}$ and $F=\int_{-\pi / 2}^{\pi / 2} T(1-T) \cos \theta_{0} d \theta_{0} / \int_{-\pi / 2}^{\pi / 2} T \cos \theta_{0} d \theta_{0}, \quad$ where 
$T=|t|^{2}$ and $G_{0}=2 e^{2} m v_{F} L_{y} / \hbar^{2}$, with $L_{y}$ denoting the width of the graphene stripe in the $y$ direction. In Fig. 5, we present the total conductance and the Fano factor as a function of Fermi energy with different lattice constants. What we should notice is that the angular-averaged conductance $G$ 's curve reaches its minimum at the DP and forms a linear cone around the $\mathrm{DP}$, and $F$ at the DP's location reaches the value of 1/3 approximately [20, 31]. From Fig. 5, we also find that the conductance and the Fano factor shift with the ratio of $w_{A} / w_{B}$ since the DP's location relies on this ratio. Therefore, this indicates that the conductance of GSL TM sequence could be modulated by the ratio of the lattice constants. In addition, we should point out that, if one compares the passing bands of Fig. 2(a) in our case with those of Fig. 2(a) in Ref. [18], one can easily find that the splitting behavior of bands in the TM sequence is very different from that in the Fibonacci case. Correspondingly, the electronic transport properties (the values of $\mathrm{G}$ and $\mathrm{F}$ ) are different from those in the Fibonacci case. These distinct differences between the TM and Fibonacci sequences will be presented in our future work.

In summary, we have studied the electronic transport properties in the graphene-based Thue-Morse aperiodic sequence. It is shown that the extra-DPs can appear under some suitable conditions. The zero- $\bar{k}$ gap associated with the DP is robust against the lattice constants and the incident angles, and the resultant controllable electron transport may facilitate the development of graphene-based electronics.

This work is supported by NSFCs (Grant. No. 11104014 and No. 61078021), Research Fund for the Doctoral Program of Higher Education of China 20110003120007 and the National Basic Research Program of China (Grant No. 2012CB921602).

[1] K. S. Novoselov, A. K. Geim, S. V. Morozov, D. Jiang, Y. Zhang, S. V. Dubonos, I. V. Grigorieva, and A. A. Firsov, Science 306, 666 (2004).

[2] Y. Zhang, Y. W. Tan, H. L. Stormer, and P. Kim, Nature (London) 438, 201 (2005).

[3] A. B. Kuzmenko, E. van Heumen, F. Carbone, and D. van der Marel, Phys. Rev. Lett. 100, 117401 (2008).

[4] F. Wang, Y. Zhang, C. Tian, C. Girit, A. Zettl, M. Crommie, and Y. R. Shen, Science 320, 206 (2008).

[5] X. Chen and J.-W. Tao, Appl. Phys. Lett. 94, 262102 (2009).

[6] A. H. Castro Neto, F. Guinea, N. M. R. Peres, K. S. Novoselov, and A. K. Geim, Rev. Mod. Phys. 81, 109 (2009).
[7] Tianxing Ma, F. M. Hu, Z. B. Huang, and Hai-Qing Lin, Appl. Phys. Lett. 97, 112504 (2010); F. M. Hu, Tianxing Ma, Hai-Qing Lin, and J. E. Gubernatis, Phys. Rev. B 84, 075414 (2011).

[8] N. M. R. Peres, Rev. Mod. Phys. 82, 2673 (2010).

[9] S. A. Wolf, D. D. Awschalom, R. A. Buhrman, J. M. Daughton, S. von. Molnár, M. L. Roukes, A. Y. Chtchelkanova, and D. M. Treger, Science 294, 1488 (2001); K. Ando, ibid. 312, 1883 (2006).

[10] R. Tsu, Superlattice to Nanoelectronics (Elsevier, Oxford, 2005).

[11] J. C. Meyer, C. O. Girit, M. F. Crommie, and A. Zettl, Appl. Phys. Lett. 92, 123110 (2008).

[12] S. Marchini, S. Günther, and J. Wintterlin, Phys. Rev. B 76, 075429 (2007).

[13] A. L. Vazquez de Parga, F. Calleja, B. Borca, M. C. G. Passeggi, Jr., J. J. Hinarejos, F. Guinea, and R. Miranda, Phys. Rev. Lett. 100, 056807 (2008).

[14] C.-X. Bai and X.-D. Zhang, Phys. Rev. B 76, 075430 (2007).

[15] M. Barbier, F. M. Peeters, and P. Vasilopoulos, Phys. Rev. B 80, 205415 (2009).

[16] L. Brey and H. A. Fertig, Phys. Rev. Lett. 103, 046809 (2009).

[17] L.-G. Wang and S.-Y. Zhu, Phys. Rev. B 81, 205444 (2010); L.-G. Wang and X. Chen, J. Appl. Phys. 109, 033710 (2011).

[18] Pei-Liang Zhao and Xi Chen, Appl. Phys. Lett. 99, 182108 (2011).

[19] C. H. Park, L. Yang, Y. W. Son, M. L. Cohen, and S. G. Louie, Phys. Rev. Lett. 101, 126804 (2008).

[20] X.-X. Guo, D. Liu, and Y.-X. Li, Appl. Phys. Lett. 98, 242101 (2011).

[21] M. Queffélec, Substitution Dynamical Systems-Spectral Analysis, Lecture Notes in Mathematics Vol. 1294 (Springer, Berlin, 1987).

[22] Z. Cheng, R. Savit, and R. Merlin, Phys. Rev. B 37, 4375 (1988).

[23] F. Axel and J. Peyriere, J. Stat. Phys. 57, 1013 (1989).

[24] J. M. Luck, Phys. Rev. B 39, 5834 (1989).

[25] X. Y. Jiang, Y. G. Zhang, S. L. Feng, K. C. Huang, Y. Yi, and J. D. Joannopoulos, Appl. Phys. Lett. 86, 201110 (2005).

[26] H. Noh, Jin-Kyu Yang, S. V. Boriskina, M. J. Rooks, G. S. Solomon, L. D. Negro, and H.Cao, Appl. Phys. Lett. 98, 201109 (2011).

[27] Nian-hua Liu, Phys. Rev. B 55, 3543 (1997).

[28] M. Kolář, M. K. Ali, and F. Nori, Phys. Rev. B 43, 1034 (1991).

[29] F. Axel, J. P. Allouche, M. Kléman, M. Mendès-France, and J.Peyrière, J. Phys. (Paris) Colloq. 47, C3-181 (1986).

[30] S. Datta, Electronic Transport in Mesoscopic Systems (Cambridge University Press, Cambridge, England, 1995).

[31] J. Tworzydlo, B. Trauzettel, M. Titov, A. Rycerz, and C. W. J. Beenakker, Phys. Rev. Lett. 96, 246802 (2006). 